\title{
SINTOMAS, FATORES DE RISCO E TRATAMENTOS DA DEPRESSÃO NA FASE DO ADOLESCER
}

\author{
Telma Sara Matos \\ (Faculdade de Filosofia, Ciências e Letras de Ribeirão Preto - USP-Ribeirão Preto - SP) \\ Ingrid Mello Pontes \\ (Universidade do Estado de Minas Gerais- Unidade Ituiutaba- MG)
}

\begin{abstract}
Resumo
Esse estudo tem como objetivo verificar o que vem sendo publicado e pesquisado nos últimos dez anos sobre a depressão durante a adolescência, assim como, também dialogar e evidenciar sobre sintomas, fatores de risco e tratamentos pela perspectiva de diferentes autores que abordem essa temática. Trata-se de uma pesquisa qualitativa, tipo descritiva, pautada na Teoria Fundamentada de Strauss e Corbin. De acordo com o método utilizado nesse estudo, foi possível listar, seguindo uma ordem cronológica de autores, o que vem sendo pesquisado sobre depressão na adolescência. Posteriormente, realizou-se uma análise qualitativa pautada em categorias para compreensão dos dados elencados. Pode-se verificar um número restrito de pesquisas que contemplam adolescência e depressão, carecendo de novos estudos que possam trazer essa correlação.
\end{abstract}

Palavras-chave: Transtorno depressivo; Adolescer; Sintomatologia; Fatores de risco; Tratamentos.

\section{Abstract \\ Symptoms, Risk Factors and Treatments of Depression in the Adolescence Phase}

This study aims to verify what has been published and researched in the last ten years about depression during adolescence, as also to discuss and evidence about symptoms, risk factors and treatments from the perspective of different authors who approached this theme. This is a qualitative research, descriptive type based on the Grounded Theory of Strauss and Corbin (2008). According to the method used in this study, it was possible to list, following a chronological order of authors, what has been researched about depression in adolescence. Subsequently, a qualitative analysis was conducted based on categories to understand the data. It can be verified a limited number of studies that contemplate adolescence and depression, lacking new studies to bring this correlation.

Keywords: Depressive Disorders; Adolescence; Symptomatology; Risk factors; Treatment.

\section{Introdução}

A depressão pode ocorrer em diversas fases do nível de desenvolvimento do indivíduo (Colavite, et. al, 2013, Bahls 2002 (a), Lima 2004). No entanto, a fase de maiores inconstâncias físicas, psíquicas 
e sociais que interferem na vida emocional da pessoa é o período da adolescência (Argimon, Terroso, Barbosa \& Lopes, 2013; Souza, Silva-Abrão \& OliveiraAlmeida, 2011; Baptista, Baptista \& Dias, 2001). A OMS salienta que a adolescência engloba a faixa etária dos 10 aos 19 anos de idade, no entanto a Organização das Nações Unidas (ONU) compreende essa faixa etária entre 15 e 24 anos (WHO, 1986) havendo, portanto, uma divergência quanto aos aspetos relacionados ao início e término da fase do adolescer.

Segundo Loures e Milagres (2009) essa fase do desenvolvimento humano tem sido alvo de debates no meio científico e acadêmico. A adolescência corresponde um período de grandes transformações na vida do indivíduo, dentre as mudanças, há o abandono ou a ressignificação de algumas posições e em suma a aquisição de novos papéis. Essa etapa do desenvolvimento refere-se a uma transição caracterizada pela demanda do “desligamento", ou seja, o adolescente necessita se afastar um pouco dos pais, para se aproximar da responsabilidade de seus próprios atos e da conquista da própria autonomia.

Ao contextualizar o período do adolescer, algumas questões emocionais devem ser consideradas, pois essa etapa corresponde a uma fase de metamorfose, iminentes mudanças e descobertas, representando um período de riscos, medos e instabilidades. Assim, a depressão no adolescente necessita da observação de determinados aspectos como, mudanças corporais, da sexualidade, oscilações hormonais e sociais (Aragão, Coutinho, Araújo \& Castanha, 2009; Barros, Coutinho, Araújo \& Castanha, 2006).

Bahls (2002a) citando em Goodyer (1996) ao abordar dados históricos, aponta que o interesse científico pela depressão em crianças e adolescentes é muito recente. Até a década de 70 acreditava-se que a depressão nessa faixa etária fosse rara ou não existisse (Bahls , 2002 b; Kent, Vostanis, \& Feehan, 1997; Larsson, Melin, Breitholtz, \& Andersson, 1991; Scivoletto, Nicastri, \& Zilberman, 1994; Shafii \& Shafii, 1992; Souza, 1984). No entanto, o Instituto Nacional de Saúde Mental dos EUA (NIMH) oficialmente reconheceu a existência da depressão em crianças e adolescentes a partir de 1975, onde as pesquisas e estudos sobre depressão nessas fases da vida têm desvelado interesse crescente no meio científico durante as duas últimas décadas (Kazdin \& Marciano, 1998; Olsson, \& von Knorring, 1997).

Esse estudo tem como justificativa o intuito de completar possíveis lacunas presentes no âmbito teórico. Tais lacunas se devem as informações de difícil acesso, visto que a bibliografia específica ao tema abordado é insuficiente, com grande 
necessidade de fomentar discussões sobre o tema.

O presente trabalho tem como objetivo verificar foi publicado e pesquisado nos últimos nove anos sobre a depressão durante a adolescência, assim como, também dialogar e evidenciar sobre conceitos, diagnósticos e tratamentos pela perspectiva de diferentes autores que abordem essa temática. Para tanto, o seguinte questionamento foi norteador desse resgate bibliográfico como: quais os diferentes sintomas, fatores de risco e tratamentos do transtorno depressivo durante o período pubertário?

\section{Contextualizando o Fenômeno}

De acordo com Biazus e Ramires (2012), um indivíduo ao entrar na adolescência, possui um corpo que naturalmente passará por diversas mudanças. Essas modificações demandam a construção de uma nova identidade, pois ocorre uma transição da infância para o adolescer. Todo esse processo vem carregado de desafios e incertezas, pois a adolescência é uma fase de sucessivas e extensas alterações, as quais operam em três âmbitos concomitantes: o psíquico, o físico e o social (Levisky, 2002; Outeiral, 2008).

Os autores Papalia e Feldman (2013) mostram que o processo do adolescer pode ser visto como a passagem entre a infância e a vida adulta, o que implica variadas transformações físicas, cognitivas e psicossociais. A puberdade seria o processo pelo qual o indivíduo atinge a maturidade sexual e a capacidade de se reproduzir. Sendo a adolescência uma longa fase na vida do indivíduo, ela ocorre com frequência nas sociedades modernas, representando uma fase de mudanças físicas, cognitivas, emocionais e sociais, constando que sua forma vai depender do contexto em que o sujeito está inserido, ou seja, de acordo com diferentes circunstâncias sociais, culturais e econômicas.

Santos, Melo Neto e Koller (2014) acrescentam que é possível entender a adolescência como uma construção social, ou seja, um período decorrente de eventos aos quais são dados significados e que são então interpretados pelos indivíduos, e também com alguns apontamentos que a sociedade destaca. A sociedade, de acordo com o contexto histórico-cultural que o sujeito está inserido, modifica suas exigências e valores.

Calligaris (2000) entende a adolescência como uma fase de se perceber adequadamente os valores transmitidos pela comunidade, ou seja, ao corpo que atingiu a maturidade e pode efetivamente dedicar-se às tarefas direcionadas por esses valores, disputando sem impeditivos com 
os outros da comunidade. Então ao haver transmissão dos principais valores sociais, tem-se um intervalo entre o início do desenvolvimento dos corpos e a permissão para então poder realizar os valores sociais, sendo essa permissão postergada para a fase adulta, tem se então um período de suspensão, chamada adolescência.

Dados do IBGE (2002) mostram que a população de adolescentes brasileira corresponde a cerca de 35 milhões, em relação à população mundial esse número aumenta para 1 bilhão de pessoas nessa faixa etária, consequentemente quase $20 \%$ da população total (Nery, Mendonça, Gomes, Fernandes, \& Oliveira, 2011).

Anteriormente, partes dos cientistas pensavam que o cérebro do adolescente estava totalmente desenvolvido, ou seja, que havia atingido seu nível máximo de maturidade. Atualmente essa perspectiva alterou devido aos estudos de imageamento cerebral, os quais demonstram uma visão anterior equivocada, revelando que o cérebro do adolescente se encontra em fase de desenvolvimento. Transformações delicadas nas estruturas cerebrais relacionadas as emoções, no julgamento, na organização do comportamento e no autocontrole, continuam o processo de desenvolvimento entre a puberdade e ao adentrar da fase adulta (Papalia \& Feldman, 2013).
A partir do século $\mathrm{XX}$, a depressão apresenta-se como uma psicopatologia, e sua manifestação na vida das pessoas estão acontecendo cada vez mais cedo. Hoje em dia, a depressão pode ser considerada como motivo de suicídio em jovens adultos e adolescentes, alinhando-se com rendimentos escolares a desejar e com o uso drogas ilícitas (Salle, Rocha, Rocha, Nunes, \& Chaves, 2012; Andrade et al., 2003; Bland, 1997; Ramchand, Griffin, Harris, McCaffrey, \& Morral, 2008).

Pode-se citar como eventos estressantes no processo do adolescer, as vivências de violência, condições de pobreza, perdas de vínculos familiares, mortes e doenças crônicas. Esses são exemplos de fatores de risco crônicos ou agudos para o surgimento de transtornos mentais, em especial se deve ponderar sobre a intensidade e frequência desses fatores (Pesce, Assis, Santos, \& Oliveira, 2004). O bullying também pode ser citado como um fator estressor, pois recentemente tem sido foco de alerta para a sociedade, pois associa-se à transtornos mentais, dentre eles a ansiedade, a depressão e o suicídio na adolescência (NASP, 2012; Kuczynski, 2014).

Segundo Argimon et al. (2013), a depressão que se apresenta na adolescência poderá trazer consequências que afetam o adequado desenvolvimento do sujeito nessa fase, como, atrapalhar o rendimento 
escolar, diminuir a autoestima, acarretando sintomas como, o desequilíbrio emocional, a irritabilidade, acessos de raiva e chances de que ocorra suicídio.

Em consonância com os dados da Organização Mundial de Saúde (OMS, 2003), cerca de $20 \%$ de crianças e adolescentes possuem algum tipo de transtorno mental. Esses transtornos podem gerar consequências nocivas para o desenvolvimento emocional dos jovens (Benetti, Pizetta, Schwartz, Hass \& Melo, 2010).

Nota-se que no decorrer da vida dos seres humanos, se tem involuntariamente a presença de acontecimentos traumáticos ou estressores que ocorrem habitualmente, mas, a negatividade, nesse aspecto se dá pelo efeito do acúmulo de situações traumáticas que aumentam a probabilidade de manifestação de psicopatologia (Dekovic, 1999; Benetti et al., 2010).

De acordo com Rutter e Taylor (2002) e Feitosa, Ricou, Rego e Nunes (2001) ao falar da saúde mental do adolescente e analisar sua origem, deve-se relacioná-los à diversos fatores como: determinismo genético, problemas cerebrais, a epilepsia, violência, luto, adversidades crônicas, fatos estressantes agudos, problemas no desenvolvimento, adoção, abrigamento, e ainda, problemas culturais e sociais que se refletem de maneira importante, no seu desenvolvimento. Segundo Avanci, Assis e Pesce (2008) citando em Rutter (2006) a hereditariedade é um fator relevante para a origem dos transtornos depressivos em adolescentes.

O Manual Diagnóstico e Estatístico de Transtornos Mentais (American Psychiatric Association - APA, 2000) não faz distinção entre os critérios de diagnósticos dos transtornos depressivos em fases variadas do desenvolvimento como: crianças, adolescentes e adultos; estando junto ao grupo dos transtornos do humor e dos transtornos bipolares, sendo especificados nos quadros do transtorno depressivo maior, do transtorno distímico e do transtorno depressivo sem diferente caracterização (Schwan \& Ramires, 2011). Os jovens geralmente possuem alterações de humor, sentimento de tristezas acentuadas, ao passo que crianças pequenas têm problemas para expressar sentimentos. Geralmente consegue-se ter uma descrição regular do psicológico na fase da adolescência (Harrington, 2002; Avanci et al., 2008).

Segundo dados da OMS (2001) e Avanci et al. (2008) não obstante nos Estados Unidos, um em cada dez jovens padece de doença mental grave, esse dado se agrava ao verificar que menos de um em cinco obteve um tratamento correto. No Brasil essa condição é complexa, pois para um país em desenvolvimento, políticas 
públicas voltadas para a saúde mental infantil, ainda carece de investimentos.

A OMS (Organização Mundial da Saúde) estipula que a depressão poderá tornar-se a segunda causa produtora de sobrecarga referente à incapacitação no ano de 2020 segundo (Bahls \& Bahls, 2002). O conceito de depressão descrito por Ballone (s/d, p.1) refere-se à depressão como "um transtorno afetivo (ou do humor), caracterizada por uma alteração psíquica e orgânica global, com consequentes alterações na maneira de valorizar a realidade e a vida" (Loures \& Milages, 2009).

Segundo Biazus e Ramires (2012) para que se tenha capacidade para compreender as diversas causas que se colocam no estabelecimento dos sintomas depressivos na fase do adolescer, faz-se necessário descrever as características do processo de adolescimento, bem como as mudanças decorrentes do processo, como: físicas, psíquicas e sociais. Vale mencionar que o grau de relevância do problema da depressão na adolescência, justamente como causa do aumento crescente de casos clínicos nessa idade, é na maior parte das vezes identificadas pelos profissionais da saúde mental (Bahls \& Bahls, 2002; Levisky, 2002).

A etiologia da depressão na adolescência que se tem conhecimento, opera por diversas causas sendo algumas delas: biológicas, genéticas, psicológicas e sociais. Discutir a respeito da depressão no adolescente implica citar uma depressão diferente, com causas e efeitos particulares, que exigem um entendimento e uma forma de intervenção especial (Biazus \& Ramires, 2012).

Sobre estes aspectos, Barros et al. (2006) e Widlöcher e Hardy (1991) e Coutinho, Gontiés, Araújo e Sá (2003) salientam que a depressão tem assumido um lugar como um dos mais perigosos problemas de saúde da sociedade atualmente, com números acentuados da doença, podendo ter incidência em todas as idades e condição socioeconômica, desconsiderando as culturas e tradições, o que traz a ela, o status de ser global, ou seja, que pode ocorrer em qualquer parte do mundo.

Sendo a depressão na infância e na adolescência uma condição duradoura, com consequências nas múltiplas funções e gerando graves danos psicossociais (Bahls, 2002b), essa psicopatologia pode tornar-se uma doença incapacitante e regular, pois de acordo com Barros et al. (2006) citando em Mirza e Michael (1996) e Walter (1996), essa enfermidade possui um alto nível de morbidade e mortalidade, tema de relevância para a saúde coletiva, mesmo não sendo corriqueiramente diagnosticada e tratada da forma correta. 
Um estudo epidemiológico de base comunitária feito no Brasil chamado Who world mental health survey initiative, enumerou cerca de $10,9 \%$ de depressão maior em adultos. Porém não se encontra um estudo estimando com o índice de transtornos depressivos na fase da adolescência tardia (idade entre 15 e 17 anos) no país. Nesse sentido, tem-se uma falta de estudos sobre depressão nesta fase peculiar (Salle et al., 2012; Kessler et al. 2010; Andrade et al., 2003).

Segundo Bahls (2002a) os sintomas do aparecimento da depressão em adolescentes estão em consonância com os apresentados pelos adultos. No entanto existem características que são peculiares do transtorno depressivo no adolescer. Adolescentes deprimidos mostram-se em especial irritáveis e instáveis, podendo apresentar crises (acessos de raiva) que aparecem na forma do indivíduo se comportar.

Como citado em Goodyer e Cooper (1993) e Avanci et al. (2008) a literatura científica evidência que uma pequena parte de crianças e adolescentes deprimidos não tiveram o experienciar de um acontecimento ou fator negativo que foi anterior à fase depressiva. Diante desses apontamentos, surgiu o interesse em verificar o que vem sendo publicado e pesquisado nos últimos dez anos sobre a depressão durante a adolescência, no intuito de debruçar sobre os aspectos teóricos na perspectiva de diferentes autores que abordem essa temática.

\section{Método}

A pesquisa bibliográfica é elaborada a partir de materiais já pesquisados e publicados, constituída em alguns casos, da exploração de livros e artigos científicos. Embora em quase todos os estudos seja exigido algum tipo de trabalho de natureza exploratória, há pesquisas desenvolvidas especialmente a partir de fontes bibliográficas ou da investigação de um estado metodológico referente ao tema pesquisado. De acordo com Gil (2008), alguns estudos exploratórios podem ser definidos como pesquisas bibliográficas.

Seguindo o exposto por Pizzani, Silva, Bello e Hayashi (2012), a pesquisa bibliográfica procura a solução de um problema (hipótese) por meio de referenciais teóricos publicados, analisando e discutindo as diferentes contribuições científicas. Esse tipo de pesquisa poderá trazer contribuições para o conhecimento sobre o que foi pesquisado, como e sob qual aspecto foi tratado certo assunto disposto na literatura científica.

Os dados trazidos por Gil (2008) demonstram ademais, que a principal vantagem da pesquisa bibliográfica consiste no fato de possibilitar ao 
investigador a cobertura de uma gama de fenômenos muito mais abrangente do que aquela que poderia pesquisar diretamente. Esta vantagem se torna particularmente importante quando o problema de pesquisa requer dados muito dispersos pelo espaço.

A análise dos dados coletados nesse estudo foi pautada em um procedimento descritivo, ancorado na Teoria Fundamentada de Strauss e Corbin (2008). Esses autores mostram um método que permite uma impecável relação entre os dados, sua referida análise relacionada à sua teoria. Ainda evidenciam que é consentido ao pesquisador que sua teoria emerja por intermédio da coleta dos dados. Segundo estes autores, a teoria provém dos dados coletados e inclina-se a se igualar mais com a realidade dos fatos, passando maior discernimento para melhor compreensão, conferindo um significativo eixo norteador para a ação do pesquisador.

De acordo com Strauss e Corbin (2008), os fundamentos teóricos que vão manifestando, relacionam-se, e esse "relacionar" de teorias é que inicia a Análise Comparativa Teórica. Estes autores salientam que, esse tipo de análise tem o benefício para estimular o pensamento do pesquisador sobre características e dimensões, com o intuito de evidenciar a amostragem teórica do estudo. Sendo que a função desse tipo de análise não visa apenas classificar e designar os dados em categorias, mas empreender um exame completo do objeto de estudo analisado, respeitando suas propriedades e dimensões. Para serem feitas comparações teóricas são fundamentais explicações mais pormenorizadas do fenômeno (Matos, 2015).

Assim, de acordo com Pizzani et al. (2012) é de extrema importância que o pesquisador faça um planejamento sistemático do processo de pesquisa, abrangendo desde a definição temática, passando pela elaboração lógica do trabalho até a decisão da sua forma de comunicação e divulgação. A revisão de literatura tem diversos objetivos, entre os quais pode-se citar: a) proporcionar um aprendizado sobre uma alguma área do conhecimento; b) auxiliar a identificação e seleção dos métodos e técnicas a serem usados pelo pesquisador; c) ofertar subsídios para a redação da introdução e revisão da literatura e redação da discussão do trabalho científico.

Para a coleta de dados desse estudo, as bases selecionadas foram: ResearchGate, Scielo (Scientific Electronic Library Online), RPMGF (Revista Portuguesa de Medicina Geral e Familiar), Periódicos Eletrônicos de Psicologia (PePSIC), Estudo geral (Universidade de Coimbra), Revista HUPE (Revista Hospital Universitário Pedro Ernesto), 
BVS MS (Biblioteca Virtual em saúde), Ares (Acervo de recursos educacionais em saúde), Biblioteca Digital de Santo André, DGS (Direção-geral da saúde), UNICEF Brasil e Mosaico (estudos em psicologia). Os descritores utilizados foram: depressão, adolescência, depressão na adolescência, doenças psíquicas durante a adolescência e problemas na atualidade enfrentados por adolescentes. Essa busca realizou-se em artigos científicos, livros, teses, relatórios e resumos executivos, obedecendo às bases de dados selecionadas, elencando referenciais teóricos publicados na última década (2009 a 2017).

Para os autores Strauss e Corbin (2008) dialogam que as pesquisas qualitativa e quantitativa precisam operar em consenso para produzir seu desenvolvimento. Se trata de um método quali-quanti, desenvolvido de maneira circular, que pode gerar a estruturação de novas técnicas teórico-metodológicas. O pesquisador deve estar aberto para perceber a manifestação dos dados, sendo que essa tarefa precisa de uma percepção “[...] nuances dos dados, tolerância para ambiguidades, flexibilidade no projeto e uma grande dose de criatividade" (Strauss \& Corbin, 2008, p. 45). Para tanto, os dados desse estudo serão organizados e apresentados de maneira a ofertar uma possibilidade de discussões em torno daquilo que emergir como significativo.
Os limites da pesquisa foram concernentes a língua em que os trabalhos foram publicados, pois, as referências bibliográficas, nas maiorias dos achados sobre o tema pesquisado estão na língua inglesa e espanhola, sendo poucos os materiais nacionais ou de língua portuguesa de cunho científico. Outro limite foi referente aos sites internacionais de banco de dados que exigiam o pagamento de uma taxa em dólares para se ter acesso a alguns achados, o que delimitou e restringiu o uso de determinadas obras, e houve a dificuldade de se encontrar bibliografias referentes ao tema da depressão no adolescente, portanto foi necessário a investigação de muitas bases de dados, para se se chegar aos 22 estudos, o critério de inclusão foi abrangendo categoria (sintomas, fatores de risco e tratamentos) no tema depressão na adolescência, data de publicação (2009 à 2017) e estar na língua portuguesa, e o de exclusão foi estar em língua inglesa e espanhola, mais de 9 anos de publicação e não aprofundar na temática da depressão no adolescente.

\section{Resultados}

Os achados correspondem a 22 referências, coletadas durante o ano de 2018, organizadas por data de publicação, título, autores, respeitando os 
delimitadores da pesquisa referentes aos sintomas, fatores de risco e tratamento do adolescente depressivo (ver Tabela 1). Foram coletados artigos científicos, livros, relatórios e resumos executivos, realizada leitura desse material, com posterior extração de dados e fichamento para a classificação do que compreendia os aspectos discutidos neste trabalho.

Tabela 1: Artigos Científicos, Livros, Teses, Relatórios e Resumos Executivos Analisados

\begin{tabular}{|c|c|c|c|}
\hline Estudo & $\begin{array}{c}\text { Data de } \\
\text { Publicação }\end{array}$ & Título & Autor (es/as) \\
\hline 1 & 2009 & $\begin{array}{l}\text { As perturbações emocionais- Ansiedade e depressão } \\
\text { na criança e no adolescente. }\end{array}$ & $\begin{array}{l}1 . \\
\text { Marques }\end{array}$ \\
\hline & 2009 & A depressão adolescente e a globalização & 2. ${ }_{\text {Milages }}$ Loures \& \\
\hline 3 & 2009 & $\begin{array}{l}\text { Recomendações para a prática clínica da saúde } \\
\text { mental infantil e juvenil nos cuidados de saúde } \\
\text { primários }\end{array}$ & $\begin{array}{l}\text { 3. Marques \& } \\
\text { Cêpeda }\end{array}$ \\
\hline 4 & 2009 & $\begin{array}{l}\text { Uma perspectiva psicossocial da sintomatologia } \\
\text { depressiva na adolescência }\end{array}$ & Aragão et al. \\
\hline 5 & 2010 & $\begin{array}{c}\text { Problemas de saúde mental na adolescência: } \\
\text { características familiares, eventos traumáticos e } \\
\text { violência }\end{array}$ & Benetti et al. \\
\hline 6 & 2011 & Depressão na infância e adolescência & $\begin{array}{l}\text { 6. Abramovitch \& } \\
\text { Aragão }\end{array}$ \\
\hline 7 & 2011 & Ansiedade e depressão na adolescência & 7. $\quad$ Brito \\
\hline 8 & 2011 & $\begin{array}{l}\text { Situação mundial da infância 2011: adolescência, } \\
\text { uma fase de oportunidades. }\end{array}$ & $\begin{array}{l}\text { 8. Fundo das } \\
\text { Nações Unidas para a } \\
\text { Infância (UNICEF) }\end{array}$ \\
\hline 9 & 2011 & Psicopatologia da Infância e da Adolescência & 9. $\quad$ Dumas \\
\hline 10 & 2011 & $\begin{array}{c}\text { Depressão em crianças: Uma breve revisão de } \\
\text { literatura }\end{array}$ & $\begin{array}{l}\text { 10. Schwan \& } \\
\text { Ramires }\end{array}$ \\
\hline 11 & 2012 & $\begin{array}{l}\text { Representações sociais e sofrimento psíquico de } \\
\text { adolescentes com sintomatologia depressiva }\end{array}$ & $\begin{array}{l}\text { Ribeiro, } \\
\text { Medeiros, Coutinho \& } \\
\text { Carolino }\end{array}$ \\
\hline 12 & 2012 & $\begin{array}{l}\text { Depressão na adolescência: uma problemática dos } \\
\text { vínculos. }\end{array}$ & $\begin{array}{l}\text { 12. Biazus \& } \\
\text { Ramires }\end{array}$ \\
\hline 13 & 2012 & $\begin{array}{l}\text { A depressão na adolescência: O risco do trauma e a } \\
\text { proteção do flourishing }\end{array}$ & Nabais \\
\hline 14 & 2012 & Adolescência, velocidade e tédio & Buchianeri \\
\hline 15 & 2013 & Depressão nos adolescentes: mito ou realidade? & $\begin{array}{l}15 . \quad \text { Resende, } \\
\text { Santos, Santos \& Ferrão }\end{array}$ \\
\hline 16 & 2013 & $\begin{array}{l}\text { Intensidade de sintomas depressivos em adolescentes } \\
\text { através da escala de depressão de Beck (BDI-II) }\end{array}$ & Argimon et al. \\
\hline 17 & 2013 & $\begin{array}{l}\text { Novas direções no tratamento da depressão na } \\
\text { adolescência: desenvolvimento e estudo de um } \\
\text { programa de intervenção psicoterapêutica }\end{array}$ & $\begin{array}{l}\text { 17. Azevedo \& } \\
\text { Matos }\end{array}$ \\
\hline 18 & 2013 & Desenvolvimento humano & $\begin{array}{l}18 . \text { Papalia \& } \\
\text { Feldman }\end{array}$ \\
\hline 19 & 2014 & $\begin{array}{l}\text { A Prevalência de transtornos mentais entre crianças e } \\
\text { adolescentes e fatores associados: uma revisão } \\
\text { sistemática }\end{array}$ & $\begin{array}{l}\text { 19. Thiengo, } \\
\text { Cavalcante \& Lovisi }\end{array}$ \\
\hline 20 & 2016 & Saúde do adolescente e a saúde da família & Oliveira et al. \\
\hline 21 & 2017 & $\begin{array}{l}\text { Depressão em Adolescentes: Revisão da Literatura e } \\
\text { o Lugar da Pesquisa Fenomenológica. }\end{array}$ & $\begin{array}{l}\text { 21. Melo, Siebra \& } \\
\text { Moreira }\end{array}$ \\
\hline
\end{tabular}


$22 \quad 2017$ Proteger e cuidar da saúde de adolescentes na atenção 22 Saúde. Ministério da

Fonte: Elaborado pela autora.

\section{Discussão}

Ao considerar o objeto de estudo o qual pretende investigar sobre as publicações dos últimos dos dez anos sobre a depressão durante a adolescência, verificou-se que aproximadamente 59,09\% dos autores apontam para os mesmos achados referentes aos aspectos que concernem a alterações do apetite e 50\% assinalam para alterações do sono. Esses autores listaram alguns sintomas correspondentes a depressão no adolescente, que pode levar a múltiplas confirmações no diagnóstico, dando maior validade a este particular aspecto.

Buchianeri (2012) dialoga que essas alterações do sono se reportam ao sujeito ter extrema sonolência durante o decorrer do dia, ou, na insônia, acorda durante a noite e não consegue dormir novamente ou não consegue pegar no sono quando se deita ou ainda, acorda bem cedo. Quanto as alterações do apetite, o autor também cita tanto a diminuição quanto o aumento do apetite, mas, a que se faz recorrente é a perda, em que o indivíduo precisa do auxílio de outras pessoas para se alimentar, ou esforçar-se para alimentar. Já no aumento de apetite o sujeito pode apresentar vontades de consumir maior quantidade de carboidratos e doces.

Aproximadamente 50\% dos autores trouxeram que o adolescente deprimido pode apresentar problemas referentes a sua autoestima, autoimagem e desvalorização, apresentando uma visão negativa do próprio corpo. Segundo a UNICEF (2011) um dos fatores que contribui para autoimagem negativa, principalmente no caso de meninas, é a propagação por meios de comunicação de um estereótipo de beleza feminina, do corpo perfeito, que não exige retoques, o que acaba trazendo comparações, de imposição de um corpo ideal, ao qual não se consegue atingir e causa como consequência sentimento de estar fora do padrão.

Em comum, os autores dos estudos $1,4,6,12,13,16,20$ e 21 apontam a desesperança como sintoma. Segundo Buchianeri (2012), os adolescentes possuem a crença irreversível de que nunca se sentirão felizes ou terão alguma forma de prazer. Outros sintomas como fadiga ou perda de energia comparecem em 50\% dos estudos, o que pode ocorrer em atividades da vida diária ou em ocupações que eram de costume. Buchianeri (2012) salienta 
ainda que mesmo na ausência de atividade física, o indivíduo ainda se queixa de cansaço, onde para realizar uma atividade simples, precisa fazer um esforço enorme. A anedonia, sintoma que pode ser explicado como a perda de prazer com a vida ou com atividades diárias, foi encontrada nas pesquisas de aproximadamente 59,09\%. Neste aspecto Buchianeri (2012) aponta para a perda de vontade de realizar atividades e passatempos prediletos, evitando com isso, as situações sociais.

Segundo Ribeiro et al. (2012) é na fase da adolescência que se estabelece o pensamento abstrato, etapa em que a pessoa passa a ter uma clareza sobre a morte, o que pode então, desencadear ideias e/ou pensamentos suicidas. Os pensamentos suicidas e tentativas de suicídios são abordados por aproximadamente $59,09 \%$ dos autores. Como exposto por Crujo e Marques (2009) este é um importante fator, indicativo de gravidade, pois nessa idade a tentativa de suicídio pode ser letal, o que implica a relevância de um correto diagnóstico e tratamento. De acordo com Brito (2011) esse comportamento suicida pode crescer nos seguintes casos: se houve a ocorrência de tentativas no passado, comorbidades psiquiátricas, uso de drogas, impulsividade e agressão, abuso físico ou sexual, ter alcance às armas letais e histórico de suicídio na família.

Por meio dessas análises, pode-se perceber que a depressão no adolescente possui características em comum com as apresentadas pelos adultos. No entanto, acrescentam-se os sintomas de irritabilidade/humor irritável, estes achados foram listados por aproximadamente $59,09 \%$ dos trabalhos. Por isso segundo Ribeiro et al. (2012) os jovens podem não estar sempre tristes, mas com grande importância irritáveis e instáveis, podendo estar presente a raiva e agressividade. O sintoma de humor depressivo/deprimido comparece nos estudos $1,3,9,10,12$, $14,15,20$ e 21 , sendo que na pesquisa de Abramovitch e Aragão (2011) é citada alteração nos sintomas dos adolescentes em relação aos adultos, com uma substituição do humor deprimido para o irritável.

De acordo com Ribeiro et al. (2012), na adolescência pode ocorrer uma alteração do comportamento como o afastamento do grupo ao qual ele se identifique, tendo como consequência desse fato, o sentimento de vazio e solidão. $\mathrm{O}$ isolamento social/solidão aparece em aproximadamente $45,45 \%$ das pesquisas. Esse isolamento também pode ser tanto em relação à família quanto de amigos/colegas. Outro aspecto observado 
foi o sentimento de tristeza, o qual está presente nos estudos 4, 6, 13, 16, 18 e 22 .

As perturbações do comportamento são apresentadas nos estudos 3, 4, 7, 11, $12,14,16,15$ e 22 , e citam a queda no desempenho escolar como uma das causas desses desajustes de conduta. Segundo Abramovitch e Aragão (2011) essa queda no rendimento escolar pode se dar devido ao sentimento de desvalia ou culpa, bloqueios no pensar ou em se concentrar.

Houveram $\quad 50 \%$ dos estudos denotando que adolescentes depressivos apresentam dificuldades em se concentrar/pensar. Scivoletto e Tarelho (2002) indicam que os adolescentes com este sintoma têm como resultado baixo rendimento escolar, diminuição da energia, falta de interesse por atividades. A culpa foi abordada por $45,45 \%$ dos autores, como citado por Resende et al. (2013), o que concerne a um sentimento prejudicial de inutilidade.

A lentificação ou agitação psicomotora são abordados por 50\% dos autores, o que segundo Sanches et al. (2005) são estes aspectos que podem revelar dois extremos: a inquietação/agitação e apatia/lentificação. No extremo desse polo de inquietação psicomotora, se tem o termo furor, e no extremo do outro polo que seria a inibição psicomotora, estaria o estupor.
As autoras Loures e Milagres (2009) apontam a globalização como influenciador no transtorno depressivo na adolescência, não sendo aludido esse excepcional achado em outras bibliografias analisadas nessa pesquisa. Estas autoras desvelam uma especial importância neste sentido, acrescentando ainda fatores relacionados aos meios de comunicação e a sociedade de consumo, como fortes influenciadores.

Quanto aos fatores de risco, o aspecto genético e hereditário, a respeito do histórico de depressão na família ou doenças psiquiátricas comparece nos estudos 3, 8, 9, 12, 15, 18, 19 e 21. A esse respeito Thiengo et al. (2014) argumentam sobre uma transmissão genética, que depende de uma combinação hereditária, ou seja, de uma interação entre um conjunto de genes, tendo ainda a participação do meio. Outros fatores como estresse/eventos

estressores/ acontecimentos desfavoráveis, aparecem nos estudos 4, 5, 11, 15, 18 e 19, referindose ao estresse como um fator que pode contribuir para um possível desenvolvimento de depressão na adolescência.

Segundo Benetti et al. (2010) o efeito dessa junção de estressores no decorrer da vida aumenta a probabilidade de psicopatologia, como exemplo, podem ser citados a violência, pobreza, relações 
familiares desfeitas, falecimentos e doenças crônicas. $\mathrm{Na}$ adolescência problemas de internalização se aglutinam às situações traumáticas, ligando-os aos problemas de perda e separações parentais.

Tal como cita os autores Resende (2013) os conflitos familiares são um fator preponderante, sendo o ambiente/estrutura familiar de grande importância para a saúde mental do adolescente. Convergindo com esses fatores de risco foram encontrados, problemas familiares, divórcio, uniões transitórias entre casais, violência na família/conjugal, mudanças e crises familiares, vinculações inseguras, rompimento de vínculo, maus-tratos, desemprego e abuso de drogas dos pais, a não expressão de sentimentos e emoções por parte dos genitores e as expectativas divergentes destes, aparecem em aproximadamente $63,63 \%$ das pesquisas.

Abusos físicos/sexuais foram apresentados nos estudos 6, 7, 8, 10, 13, 18 e 19 Nabais (2012) explica o abuso físico como algo que machuque a pessoa, batendo e agredindo de diferentes formas. A negligência/abandono, foi mostrado nos estudos 7 e 13, em que foi salientando por Nabais (2012) que essa negligência, possui o significado de não se fazer presente na relação entre um responsável e o adolescente, havendo uma falta ou ausência das necessidades básicas (educacional, alimentar, higiênica, afetiva ou sanitária).

Problemas escolares foram apontados nos estudos 3, 8, 10, 11, 15 e 22. Segundo Schwan e Ramires (2011), se reproduz em um déficit escolar, relacionado a não conseguir acompanhar os colegas de classe da mesma faixa etária. O bullying apareceu na pesquisa 10 , sendo considerado no ambiente escolar, como uma rejeição por parte dos colegas.

Houve ainda alguns fatores de risco, que apareceram em menor número nos estudos como: exclusão social (4 e 8), autoestima/autoimagem ruim (4 e 10), perdas (4 e 15), situação de acolhimento (5), eventos traumáticos (5, 7 e 11), violência escola/comunidade (5, 8, 13, 19 e 21), falta de diálogo (5), baixa renda/pobreza (6, 8 e 21), más condições de moradia

analfabetismo/desvantagem educacional/dificuldade escolar (6, 8 e 15), pouco acesso à saúde (6), fatores neurobiológicos (9 e 19), psicológicos e sociais (9), TDAH (10), uso precoce de álcool (10,18 e 21), atividade sexual ligada ao abuso de drogas/álcool (18), doenças psiquiátricas (8 e 15), crises humanitárias/desastres naturais e conflitos armados (8), estigma dirigido (8), violação direitos humanos (8), não tratamento psicológico na infância (13 e 15), 
problemas com pares (15), doenças crônicas (18) e comportamento suicida (7).

No que tange ao tratamento, os estudos apontam para a promoção de saúde (13), apoios sociais (1 e 21), tratamento especializado/ aumento de atendimento especializado (19 e 8), psicoterapia (1, 18, 17, 15, 6, 8 e 10), medicação $(1,18,17,15$, 6, 7 e 10), hospitalização para os que tem resposta negativa por tratamento ambulatorial, dependentes de substancias químicas, psicóticos e tendência suicida (18), intervenção psicossocial (15), combinação de terapias (15 e 18), promoção de saúde (13), ações na saúde e na educação escolar (1 e 4), orientação de pais e escola (6), envolvimento da família (1, 7 e 8), programas psicossociais na escola (8), orientação de apoio (8).

Portanto, o aspecto mais comum como forma de tratamento nos estudos são a psicoterapia e a medicação. A maioria dos estudos trouxeram formas de tratamento diferentes, acrescentando aspectos e áreas que influam nos sintomas do adolescente depressivo (escola e família). Porém, segundo Schwan e Ramires (2011) há uma divergência quanto às formas de tratamentos eficazes, pois existem poucas pesquisas sobre a relevância da participação dos pais e família no tratamento psicoterapêutico.

Segundo Azevedo e Matos (2013) a psicoterapia diz respeito ao tratamento geralmente indicado à casos leves e moderados, já a psicofarmacoterapia, é indicada para casos graves e em indivíduos que não reagem a psicoterapia. Schwan e Ramires (2011) descrevem que o melhor tratamento tanto para crianças quanto adolescentes (nos casos leves e moderados) é a psicoterapia, porém nos casos graves mencionam a combinação desta, com a medicação. Pontuam que as intervenções podem ser com o indivíduo, em grupo ou com a família.

A UNICEF (2011) cita como uma das formas de tratamento útil para saúde mental dos adolescentes, o envolvimento da família e programas psicossociais nas escolas. Ribeiro et al. (2012) falam da importância do afeto, no tratamento da depressão em adolescentes, não só da família, mas também do meio social.

Precisa-se, portanto, segundo Brito (2011) de um tratamento plurimodal, mas que inclua a psicoterapia, medicação e a ajuda da família, considerando que a psicoterapia é de primeira linha e nos casos de medicação, a Sertralina, o Escitalopram, a Paroxetina, a Fluvoxamina e a Fluoxetina, são indicados.

Os sintomas podem ter certa correspondência com os do adulto, acrescentando-se, todavia, a modificação do humor depressivo por irritável. Nos fatores de risco têm-se tanto os aspectos internos quanto externos, como 
mencionam Melo et al. (2017), apontando que a família é muito importante na formação e sustentação da personalidade do adolescente.

Infelizmente no tratamento da depressão para adolescentes, muitas vezes utiliza-se as mesmas formas de intervenção, assim como é realizada nos adultos. Ou seja, tem-se apenas uma adaptação do tratamento, o que pode não contemplar as características peculiares dessa fase do desenvolvimento humano, como a síndrome normal da adolescência. Essa síndrome de acordo com Knobel (1981) relaciona-se a uma procura pela identidade, inclinação ou gosto por grupos, impasses religiosos, urgência por usar da fantasia e intelectualização, pensamento voltado para o imediato, desenvolvimento sexual que começa no autoerotismo e vai até a heterossexualidade genital adulta, oposições na conduta no que se refere a ação, atitude social reinvidicatória, afastamento dos pais, humor e ânimo instáveis.

Este estudo procurou trazer um olhar científico sobre as questões relacionadas à depressão na adolescência, com base em referenciais teóricos. Ao considerar que nos dias atuais não há a propagação de muitas informações sobre o tema, ressalta-se que esse estudo procurou o aporte científico, para desvelar sobre a depressão no adolescente, e quão esse assunto comparece no meio acadêmico.

\section{Considerações Finais}

Diante do recurso metodológico utilizado nesse estudo, foi possível listar, seguindo uma ordem cronológica de autores, o que vem sendo pesquisado sobre a depressão na adolescência, considerando os sintomas, fatores de risco e tratamentos. Posteriormente realizou-se uma análise qualitativa e quantitativa por meio de categorias (sintomas, fatores de risco e tratamentos) para uma melhor compreensão dos dados elencados.

$\mathrm{Na}$ análise dos 22 estudos, pode-se observar que a maioria apresentou aspectos relacionados aos sintomas como irritabilidade, anedonia, baixa autoestima, ideação/tentativa suicida, desesperança, fadiga, humor depressivo, isolamento, tristeza, perturbações do comportamento e dificuldades de pensar ou de se concentrar. Nos fatores de risco, a maioria citou o aspecto genético, eventos estressores, conflitos familiares, abusos físicos/sexuais, abandono, negligência e problemas escolares. Por último nos tratamentos, houve maior concordância entre a psicoterapia para casos leves e moderados, e a medicação para casos graves.

Pode-se verificar que esse tema necessita de outras pesquisas, pois ao 
considerar a adolescência, além desta envolver uma fase repleta de mudanças, muitas vezes essa etapa da vida é considerada secundária pelos pesquisadores, que tendem focar seus estudos contemplando apenas as fases da infância e adulta. No que tange aos materiais científicos reportando à depressão, existem em grande quantidade, no entanto, esses materiais não aprofundaram seus estudos especificando o adolescente em questão.

O presente trabalho procurou evidenciar aspectos ligados ao transtorno depressivo no adolescente com o intuito de elucidar para os profissionais da área de saúde mental, principalmente da Psicologia, possibilitando um melhor entendimento do individuo e um tratamento psicoterápico que considere a fase de desenvolvimento em questão.

\section{Referências}

Abramovitch, S. \& Aragão C. (2011). Depressão na infância e adolescência. Revista Hospital Universitário Pedro Ernesto.10(2):41-46. Recuperado em 10, Abril, 2018, de http://revista.hupe.uerj.br.

American Psychiatric Association. (2000). DSM-IV-TR, Manual Diagnóstico e Estatístico de Transtornos Mentais. Porto Alegre: Artes Médicas.

Andrade L., Caraveo-Anduaga J., Berglund P., Bijl R.V, De Graaf R. \& Vollebergh W. The epidemiology of major depressive episodes: results from the International Consortium of Psychiatric Epidemiology (ICPE) Surveys. Int J Methods Psychiatr Res. 2003;12(1):321. https://doi.org/10.1002/mpr.138.

Aragão, T. A., Coutinho, M. D. P. D. L., Araújo, L. F. D., \& Castanha, A. R. (2009). Uma perspectiva psicossocial da sintomatologia depressiva na adolescência. Ciência \& Saúde Coletiva, 14, 395-405. Recuperado em 14, Abril, 2018 de http://www.scielo.br. https://doi.org/10.1590/S1413-81232009000200009.

Argimon, I. L., Terroso, L. B., Barbosa, A. S., \& Lopes, R. F. (2013). Intensidade de sintomas depressivos em adolescentes através da escala de depressão de Beck (BDI-II). Boletim Academia Paulista de Psicologia, 33(85), 354-372. Recuperado em 19 de Abril, 2018, de: http://pepsic.bvsalud.org/scielo.phpt. 
Avanci J. Q, Assis S. G \& Pesce R. P. (2008). Depressão em crianças: uma reflexão sobre crescer em meio à violência. Rio de Janeiro: Fiocruz/Ensp/Claves/Cnpq. pp. 11-74.

Azevedo, A., \& Matos, A. P. (2013). Novas direções no tratamento da depressão na adolescência: desenvolvimento e estudo de um programa de intervenção psicoterapêutica. Livro de Atas do VIII Simpósio Nacional de Investigação em Psicologia , 816-824. Recuperado em 10 Março, 2018, de: https://estudogeral.sib.uc.pt

Bahls, S. C. (2002a). Aspectos clínicos da depressão em crianças e adolescentes. Jornal de Pediatria, Curitiba, 78(5), 359-366. https://doi.org/10.1590/S0021-75572002000500004.

Bahls, S. C. (2002b). Epidemiologia de sintomas depressivos em adolescentes de uma escola pública em Curitiba, Brasil. Revista Brasileira de Psiquiatria, 24 (2), 33-44.

Bahls, S. C., \& Bahls, F. R. C. (2002). Depressão na adolescência: características clínicas. Interação em Psicologia, 6(1). https://doi.org/10.5380/psi.v6i1.3193.

Ballone, G. J. O que é depressão. s/d. Recuperado em 20 Março, 2018, de https:www.gballone.sites.uol.com

Baptista, M. N., Baptista, A. S. \& Dias, R. R. (2001). Estrutura e suporte familiar como fatores de risco na depressão de adolescentes. Psicologia: Ciência e Profissão. V. 21(n.2), 52-61. Recuperado em 10 Março, 2018, de: http://www.scielo.br. https://doi.org/10.1590/S1414-98932001000200007.

Barros, A. R., Coutinho, M. L., Araújo, L. F., \& Castanha, A. R. (2006). As representações sociais da depressão em adolescentes no contexto do ensino médio. Estudos de Psicologia (Campinas), 23(1), 19-28. Recuperado em 10 March, 2018, de: http://www.scielo.br. https://doi.org/10.1590/S0103-166X2006000100003.

Benetti, S. P., Pizetta, A., Schwartz, C. B., de Azevedo Hass, R., \& Melo, V. L. (2010). Problemas de saúde mental na adolescência: características familiares, eventos traumáticos e violência. Psico-usf, 15(3), 321-332. Recuperado em 10 Março, 2018, de http://www.scielo.br. https://doi.org/10.1590/S1413-82712010000300006. 
Biazus, C. B., \& Ramires, V. R. (2012). Depressão na adolescência: uma problemática dos vínculos. Psicologia em Estudo, 17(1), 83-91. Recuperado em 10 Março, 2018, de: http://www.scielo.br. https://doi.org/10.1590/S1413-73722012000100010.

Bland R. C. (1997). Epidemiology of affective disorders: a review. Can J Psychiatry. 1997;42(4):367-77. https://doi.org/10.1177/070674379704200403.

Brito, I. (2011). Ansiedade e depressão na adolescência. Revista Portuguesa de Clínica Geral, 27(2), 208-214. Recuperado em 10 April, 2018, de http://www.scielo.mec.pt/ https://doi.org/10.32385/rpmgf.v27i2.10842.

Buchianeri, L. G. C. (2012). Adolescência, velocidade e tédio. São Paulo: Ed. Unesp. Recuperado em 15, Abril, 2018 de http://www2.santoandre.sp.gov.br/

Calligaris, C. (2000). A Adolescência. São Paulo: Publifolha.

Colavite, J., Silva, F. F., Garbi, J. P., Silva, M. O., Ribeiro, R. A., \& Cardoso, H. F. (2013). Depressão: crianças também sofrem com essa doença. Psicólogo Informação, 17(17), 123-131. https://doi.org/10.15603/2176-0969/pi.v17n17p123-131.

Coutinho, M. P. L., Gontiés, B., Araújo, L. F., \& Sá, R. C. N. (2003). Depressão um sofrimento sem fronteira: representações sociais entre crianças e idosos. Psico-USF, 8 (2), 182-191. https://doi.org/10.1590/S1413-82712003000200010.

Crujo, M., \& Marques, C. (2009). As perturbações emocionais - Ansiedade e depressão na criança e no adolescente. Revista Portuguesa de Medicina Geral e Familiar, 25(5), 57682. Recuperado em 10 April, 2018, de http://www.rpmgf.pt. https://doi.org/10.32385/rpmgf.v25i5.10675.

Deković, M. (1999). Risk and protective factors in the development of problem behavior during adolescence. Journal of youth and adolescence, 28(6), 667-685. https://doi.org/10.1023/A:1021635516758.

Dumas, J. (2011). Os transtornos de humor. In F. B. Assumpção Jr. (ed.). Psicopatologia da infância e da adolescência (3 a edição, pp. 335-446). Bruxelas, Artmed. 
Feitosa, H. N, Ricou M., Rego, S., \& Nunes, R. (2011). A saúde mental das crianças e dos adolescentes: considerações epidemiológicas, assistenciais e bioéticas. Revista Bioética, 19(1).

Gil, A. C. (2008). Métodos e Técnicas de Pesquisa Social. $6^{\text {a }}$ Ed. São Paulo: Atlas.

Goodyer I. M. (1996). Physical symptoms and depressive disorder in childhood and adolescence. $\quad J$ Psychosom Res 1996;41(5):405-8. https://doi.org/10.1016/S00223999(96)00154-7.

Goodyer, I., Ashby, L., Altham, P.M.E., Vize, C., \& Cooper, P.J. (1993). Temperament and major depression in 11-16 year-olds. Journal of Child Psychology and Psychiatry; 34: 1409-1423. https://doi.org/10.1111/j.1469-7610.1993.tb02099.x.

Harrington, R.C. (2002). Affective Disorders. In: Rutter, M.; Taylor, E. Child and Adolescent Psychiatry. USA: Blackwell Publishing. p.463-485.

Harrington, R.C., Fudge, H., Rutter, M., Pickels, A., \& Hill, J. (1990). Adult outcomes of childhood and adolescent depression: II. Psychiatric status. Archives of General Psychiatry; 47: 465-473. https://doi.org/10.1001/archpsyc.1990.01810170065010.

Instituto Brasileiro de Geografia e Estatística. (2002). Estatísticas da saúde: assistência médico-sanitária. Recuperado em 20 Março, 2018, de http://www.ibge.gov.br/estatísticas.

Kazdin A. E, \& Marciano P. L. (1998). Childhood and adolescent depression. In: Mash E, Barkley R, editores. Treatment of Childhood Disorders. 2a ed. New York: The Guilford Press.

Kent L., Vostanis P., \& Feehan C. (1997). Detection of Major and Minor Depression in Children and Adolescents: Evaluation of the Mood and Feelings Questionnaire. J Child Psychol Psychiatry; 38(5):565-73. https://doi.org/10.1111/j.1469-7610.1997.tb01543.x.

Kessler, R. C. (2012). The costs of depression. Psychiatric Clinics of North America, 35, 114. https://doi.org/10.1016/j.psc.2011.11.005.

Kessler R. C., Birnbaum H.G., Shahly V., Bromet E., Hwang I., \& McLaughlin, K.A. (2010). Age differences in the prevalence and co-morbidity of DSM-IV major depressive 
episodes: results from the WHO World Mental Health Survey Initiative. Depress anxiety. 2010;27(4):351-64. https://doi.org/10.1002/da.20634.

Knobel, M. (1981). A síndrome da adolescência normal. In: Aberastury, A., Knobel, M. Adolescência normal: um enfoque psicanalítico. Porto Alegre: Artes Médicas, p. 24-59.

Kuczynski, E. (2014). Suicídio na infância e adolescência. Psicologia USP, 25(3), 246-252. Recuperado em 10 Março, 2018, de http://www.scielo.mec.pt/ . https://doi.org/10.1590/0103-6564D20140005.

Larsson, B., Melin, L., Breitholtz, E. \& Andersson G. (1991). Short-term stability of depressive symptoms and suicide attempts in Swedish adolescents. Acta Psychiatr Scand.;83:385-90. https://doi.org/10.1111/j.1600-0447.1991.tb05561.x.

Levisky, D. L. (2002). Depressões narcísicas na adolescência e o impacto da cultura. Psychê, 6(10), 125-136.

Lima, D. (2004). Depressão e doença bipolar na infância e adolescência. J. Pediatr. (Rio Janeiro), v. 80, n. 2, p. 11-20, 2004. Recuperado em 20, Abril, 2018, de http://www.scielo.br. https://doi.org/10.1590/S0021-75572004000300003.

Loures, N. R. P., \& Milagres, A. F. (2009). A depressão adolescente e a globalização. Mosaico: Estudos em Psicologia, 3(1). Recuperado em 10 de Abril, 2018, de https://seer.ufmg.br/.

Marques, C. \& Cêpeda, T. (2009). Recomendações para a prática clínica da coordenação nacional para a saúde mental infantil e juvenil nos cuidados de saúde primários. Portugal, Ministério da Saúde, Alto Comissariado da Saúde, Coordenação Nacional para a Saúde Mental. 52p. Recuperado em 24, Março, 2018, de https://www.dgs.pt/ https://doi.org/10.32385/rpmgf.v25i5.10674.

Matos, T. S. Q. (2015). Formação de professores: diálogos entre a Educação Física e a Psicologia. 2015. Dissertação (Mestrado em Educação) - Programa de Pós Graduação em Educação, Universidade Federal do Triângulo Mineiro, Uberaba.

Melo, A., Siebra, A., \& Moreira, V. (2017). Depressão em Adolescentes: Revisão da Literatura e o Lugar da Pesquisa Fenomenológica. Psicologia: Ciência e 
Profissão, 37(1), 18-34. Recuperado em 20, March, 2018, de http://www.scielo.br. https://doi.org/10.1590/1982-37030001712014.

Ministério da Saúde. (2017). Proteger e cuidar da saúde de adolescentes na atenção básica. Brasil. Recuperado em 26 Março, 2018, de http://bvsms.saude.gov.br.

Mirza, K., \& Michael, A. (1996). Major depression in children and adolescents. British Journal Hospital Medical, 55 (1-2), 57-61.

Nabais, M. E. C. (2012). A depressão na adolescência: o risco do trauma e a proteção do flourishing. Tese de mestrado, Estudo Geral, Universidade de Coimbra, Portugal. Recuperado em 30 de Março, 2018, de https://estudogeral.sib.uc.pt.

National Association of School Psychologists. (2012). Bullying prevention and intervention in schools. Bethesda, MD: Author.

National Survey on Drug Use and Health (NSDUH). (2008). Major depressive episode among youths aged 12 to 17 in the U.S.: 2004 to 2006. The NSDUH Report. Author.

Nery, I. S., Mendonça, R. M., Gomes, I. S., Fernandes, A. N. N., \& Oliveira, D. C. (2011). Reincidência da gravidez em adolescentes de Teresina, PI, Brasil. Rev. bras. enferm. Feb; 64( 1 ): 31-37. Recuperado em 20, March, 2018, de: http://www.scielo.br/ https://doi.org/10.1590/S0034-71672011000100005.

Nico, T., Leonardi, J. L.\& Zeggio, L. (2015). A Depressão como Fenômeno Cultural da Sociedade Pós-moderna - Parte I: Um Ensaio Analítico-Comportamental dos Nossos Tempos. São Paulo: Creative Commons.

Oliveira, A., Raymundo, C., Mathias, C., Grossman, C., Bastos, F., d'Abreu, H., Felipe, I., Antunez, M., Albuquerque, V. (2016). Saúde do adolescente e a saúde da família Universidade Federal do Maranhão. UNA-SUS/UFMA. São Luís: EDUFMA. 88 p.: il. (Cadernos de Saúde da Família, n. 6). Recuperado em 20, Março, 2018 de https://ares.unasus.gov.br/.

Olsson, G., von Knorring, A-L. (1997). Depression among Swedish adolescents measured by the self-rating scale Center for Epidemiology Studies - Depression Child (CES-DC). Eur Child Adolesc Psychiatry; 6:81-7. https://doi.org/10.1007/s007870050012. 
Organização mundial da saúde (OMS). (2001). Saúde Mental: Nova Concepção, Nova Esperança. Geneva: WHO.

Organização mundial de saúde (OMS). (2018). Depression. Recuperado em 20 April, 2018, de: http://www.who.int/mediacentre/factsheets/fs369/en/

Outeiral, J. (2008). Adolescer (3a ed.). Rio de Janeiro: Revinter

Papalia, D. E., \& Feldman, R. D. (2013). Desenvolvimento Físico e cognitivo na adolescência. In Silva, M., Lima, M. Pinheiro, O., Maciel Jr., P. (Ed.). Desenvolvimento humano. (12a edição, pp. 384-419). Porto Alegre, Artmed.

Pesce, R. P., Assis, S. G. D., Santos, N., \& Oliveira, R. D. (2004). Risco e proteção: em busca de um equilíbrio promotor de resiliência. Psicologia: teoria e pesquisa, 20(2), 135-143. https://doi.org/10.1590/S0102-37722004000200006.

Pizzani, L., Silva, R., Bello, S., \& Hayashi, M. (2012). A arte da pesquisa bibliográfica na busca do conhecimento. RDBCI: Revista Digital de Biblioteconomia e Ciência da Informação, 10(2), 53-66. https://doi.org/10.20396/rdbci.v10i1.1896.

Ramchand, R., Griffin, B. A., Harris, K. M., McCaffrey, D. F., \& Morral, A. R. (2008). A prospective investigation of suicide ideation, attempts, and use of mental health service among adolescents in substance abuse treatment. Psychol Addict Behav. ;22(4):524-32. https://doi.org/10.1037/a0012969.

Resende, C., Santos, E., Santos, P., \& Ferrão, A. (2013). Depressão nos adolescentes: mito ou realidade?. Nascer e Crescer, 22(3), 145-150. Recuperado em 13, Março, 2018, de http://www.scielo.mec.pt

Ribeiro, K., Medeiros, C., Coutinho, M., \& Carolino, Z. (2012). Representações sociais e sofrimento psíquico de adolescentes com sintomatologia depressiva. Psicologia: teoria e prática, 14(3), 18-33. Recuperado em 13, Março, 2018, de http://pepsic.bvsalud.org/.

Rutter, M., Taylor, E. (2002). Child and adolescent psychiatry. Oxford: Blackwell Publishing.

Rutter, M. (2006). Genes and Behavior. USA: Blachwell-Publishing. 
Salle, E., Rocha, N. S., Rocha, T. S., Nunes, C., \& Chaves, M. L. (2012). Escalas psicométricas como instrumentos de rastreamento para depressão em estudantes do ensino médio. Revista de Psiquiatria Clínica, 39(1), 24-27. https://doi.org/10.1590/S0101-60832012000100005.

Sanches, M., Marques, A. P., Ortegosa, S., Freirias, A., Uchida, R., \& Tamai, S. (2005). O exame do estado mental. É possível sistematizá-lo?. Arquivos Médicos dos Hospitais e da Faculdade de Ciências Médicas da Santa Casa de São Paulo, 50(1), 18-23.

Santos, E. C., Melo Neto, O. C., Koller, S. (2014). Adolescentes e Adolescências. In Habigzang, L. F., Diniz, E., \& Koller, S. H. (Org.). Trabalhando com adolescentes: Teoria e intervenção psicológica. Porto Alegre, RS: Artmed

Schwan, S., \& Ramires, V. (2011). Depressão em crianças: Uma breve revisão de literatura. Psicol. Argum, Curitiba, v. 29, n. 67, p .457-468, dez. Recuperado em 15, Abril, 2018 de https://www.researchgate.net/ https://doi.org/10.7213/psicolargum.v29i67.20307.

Scivoletto, S., Nicastri S., \& Zilberman M. L. (1994). Transtorno depressivo na adolescência: diagnóstico e tratamento. RBM; 51(9): 1211-28.

Scivoletto, S., \& Tarelho, L. G. (2002). Depressão na infância e adolescência. RBM rev. bras. med, 59(8), 555-558.

Shafii, M., \& Shafii, S. L. (1992). Clinical Manifestations and Developmental Psychopathology of Depression.In: Shafii M, Shafii SL, editores. Clinical Guide to Depression in Children and Adolescents. Washington: American Psychiatric Press. p.342.

Souza, P. R. (1984). Depressão endógena em criança de oito anos - relato de caso. Revista da Associação Brasileira de Psiquiatria; 6(21):67-9.

Souza, E.M., Siva-Abrão, F.P \& Oliveira-Almeida, J. (2011). Desigualdade social, delinquência e depressão: um estudo com adolescentes em conflito com a lei. Revista de Salud Publica. V. 13 (n.1), 13-18.

Strauss, A. \& Corbin, J. (2008). Pesquisa qualitativa: técnicas e procedimentos para o desenvolvimento de teoria fundamentada. 2. ed. Porto Alegre: Artmed. 288 p. 
Tavares, L. A. T. (2010). A depressão como "mal-estar" contemporâneo: medicalização e (ex)-sistência do sujeito depressivo. São Paulo, Editora da Unesp/Cultura Acadêmica. Recuperado em 30 Março, 2018, de: http://books.scielo.org/id/j42t3 https://doi.org/10.7476/9788579831003.

Thiengo, D., Cavalcante, M., \& Lovisi, G. (2014). Prevalência de transtornos mentais entre crianças e adolescentes e fatores associados: uma revisão sistemática. Jornal Brasileiro de Psiquiatria, 63(4), 360-372. Recuperado em 20, Março, 2018, de http://www.scielo.br/. https://doi.org/10.1590/0047-2085000000046.

UNICEF. (2011). Situação mundial da infância 2011: adolescência, uma fase de oportunidades. Brasília, DF: Autor. Recuperado em 27, Abril, 2018, de https://www.unicef.org/brazil/

Walter, G. (1996). Depression in adolescence. Australian Family Physician, 25 (10), 15751582.

WHO, World Health Organization. (1986). Young People's Health - a Challenge for Society. Report of a WHO Study Group on Young People and Health for All. Technical Report Series 731. Geneva: WHO.

Widlöcher, D., \& Hardy, M. C. (1991). La Dépression. Paris: Overture Medicale.

World Health Organization. (2003). Caring for children and adolescents with mental disorders. Setting WHO directions. Geneva: World Health Organization.

\section{As autoras:}

Telma Sara Matos, doutoranda no Programa de Pós-graduação da Faculdade de Filosofia, Ciências e Letras de Ribeirão Preto -USP-Ribeirão Preto. Possui Mestrado em Educação pela Universidade Federal do Triângulo Mineiro (2015). Possui graduação em Formação e Bacharelado em Psicologia pelo Centro Universitário do Triângulo (2008) e graduação em Licenciatura Plena em Educação Física pela Universidade Federal de Uberlândia (1998). telmapsicoesporte@gmail.com

Ingrid Mello Pontes, possui Bacharelado em Psicologia pela Universidade do Estado de Minas Gerais (2018). ingridmellpon@gmail.com

Recebido em: 17/12/2018

Aprovado em: 25/05/2019 